\title{
Network communication and identification of the cyber-physical systems
}

\author{
Anatoly V. Shukalov ${ }^{1}$, Igor O. Zharinov ${ }^{1}$ and Oleg O. Zharinov ${ }^{2}$ \\ ${ }^{1}$ Faculty of Information Security and Computer Technologies, ITMO University, 49, Kronverksky Av., Saint \\ Petersburg, 197101, Russia \\ ${ }^{2}$ Department of Problem-Oriented Computing Complexes, Saint Petersburg State University of Aerospace \\ Instrumentation, 67, Bolshaya Morskaia str., Saint Petersburg, 190000, Russia
}

\begin{abstract}
Cyber-physical system net communication and identification are connection and information theory elements, which let define data transmission dynamic routes. The net commutation is done with gate system, concentrators and routers to provide distributed connection of close and far range radius for the production equipment. The net commutation is done during the connection system different topologies virtual construction for the production operation processes. The net identification is done with a number of physical, logical and information attributes, which in their values or properties represent clearly a connection system industrial device. The different options how to address a device and meta-data are shown, which are important for net identification procedure. There are information net dynamic topology options proposed, which represent the cyber-production operation environment using equipment identification principles for technological and control data routing and processing.
\end{abstract}

\section{Keywords}

Network, communication, cyber-physical system, Industry 4.0

\section{Introduction}

The cyber-physical system (CPS) information net topology basic property is its physical and virtual connection channels architecture resilience, which are used to transmit messages and cloud servers agents communication $[1,2]$. The main inter-net cyber-physical production (CPP) exchange participant is a CPS containing telecommunication units of reception and transmission and data processing $[3,4]$. A combination of CPSs forms a complicated production segment containing information, measuring and controlling components, which are for product manufacturing in the company operation net $[5,6]$.

The CPP technological tasks solution is done as a plurality of net technologies of feeble link establishing CPS telecommunication connections to transmit messages of different priorities for permissible time [7, 8]. A CPP trouble area is the information net dynamic topologies to define the CPS self-organization property with different options of their usage $[9,10]$.

The CPP traffic is a combination of [11, 12]:

- the CPS out-coming messages packages, which inform the cloud applications of the processes and equipment actual conditions;

- the CPS incoming messages packages receiving control commands.

The packages useful load is a CPS control device element containing net agents identification means, means to measure and transmit technological data to the link channel, microprocessor messages and executive mechanisms processing means, which position is regulated remotely $[13,14]$.

III International Workshop on Modeling, Information Processing and Computing (MIP: Computing-2021), May 28, 2021, Krasnoyarsk, Russia

EMAIL: aviation78@mail.ru (Anatoly Shukalov); mpbva@mail.ru (Igor Zharinov); zharinov73@inbox.ru (Oleg Zharinov) ORCID: 0000-0003-0283-5207 (Anatoly Shukalov); 0000-0003-2508-5939 (Igor Zharinov); 0000-0003-1219-8205 (Oleg Zharinov)

(c) (i) (C) 2021 Copyright for this paper by its authors.

Use permitted under Creative Commons License Attribution 4.0
CEUR Workshop Proceedings (CEUR-WS.org) 


\section{The CPS net identification}

To improve industrial object production indicators means to build an effective CPS interaction scheme. The Industry 4.0 CPP technology more important status is its Internet of Things (IoT) net using mechanisms of multi-channel data transmission and reception and packages routing compatible with any link protocols types. The IoT wireless nets subject area includes logical and physical message transmission aspects and supports any CPS communication models with connection control function in the available address company space [15].

Computer nets technologies and industrial equipment integrated calculation systems unification made actual the new type of production means synthesis problem being controlled with radio technologies. CPS autonomous devices connected to the net make some production operations and transform radio frequency packages into IP (Internet Protocol)-traffic in the CPS addressing model level. The CPS identification control system provides only one way to address them and initializes equipment initial state inside the company information net with some machine decoders and special software applications, which convert the connection protocols.

The perspective things for CPP implementation are some competing net automatic identification systems, which are potentially useful for CPS peripheral telecommunications:

- CPS identification based on unique MAC (Media Access Control)-addresses decoding established by physical equipment designer in the net adapter;

- CPS identification based on wireless message demodulation containing unique within the company address space logical IP;

- CPS identification based on MSISDN (Mobile Subscriber Integrated Services Digital Number), IMEISV (International Mobile Terminal Identity \& Software Version Number), IMSI (International Mobile Subscriber Identity) or IMEI (International Mobile Equipment Identity) numbers, which format let clearly associate industrial equipment sample with inner-net domain names;

- CPS identification based on meta-data attributively characterized in the machine information level available for installed software and machine-ware components net analysis (software versions, operation system type, electronic units devices nomenclature and other) and other.

The CPP net CPS stable identification is done in business analytics platform having attributive properties registers, addresses tables and equipment protocol to guarantee agent clear identification in the industrial automatics application field. New ways of CPS net interaction in fact are related to the company address space are based on telecommunication technology development theory and are justified with automatic production systems functionality specifics.

The Industry 4.0 technologies multiplicative influence for a CPP provides the industrial object critically crucial processes stable control where the CPS net component is an intellectual instrument. The CPP architecture integration feature is to apply machine telemetry engaging all variety of information protocols for a net structure organization (configuration) through which the technological data and control data are being translated. Connection among CPS devices forms the CPP control loop closed state when the reverse chains are defined with commutative congregations, which have the dynamic packages transmission routes and net services.

\section{The CPS information net topology}

Incoming and out-coming packages connection lines, which are used to connect a CPS to the net and the way to transport the messages to the agents depend on the topology features, which are CPP dynamic structures in their role. The CPP information net dynamic topology options are given in Figure $1:$. 


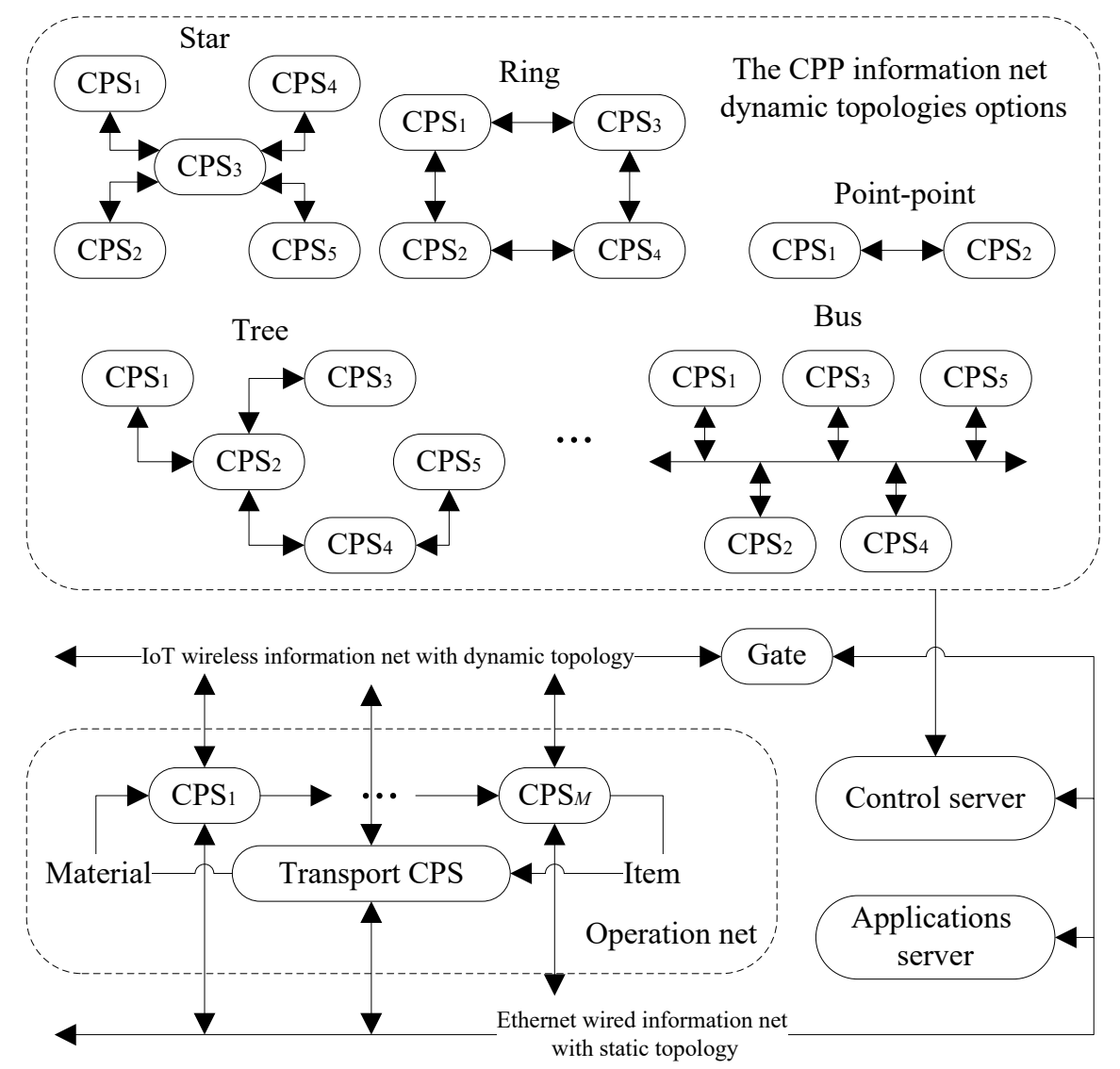

Figure 1: The CPP information net dynamic topology options

A connection session done with end CPS devices could be done in the following relatively simple information net topology configurations:

- the structure communication «point-point», which are basic for two-direction CPS connection are normally installed in a company in the proximity to each other. The CPS contact is done without intermediary gates of low connection radius. The topology option is a functional net unit, which is a part of more complicated commutation types;

- the structure communication «ring», which is for the traffic exchange in an isolated CPP segment (CPS group). The CPS ring net feature is its connection channel low passing through ability justified with subsequent packages transmission through all agents structure from the message sending unit to the reception one. The message translation long state depends on the CPS number (net agents), which passes through the traffic. The structure connection control is directed to the data transmission minimal net way search;

- the structure communication «star» is for CPS control with the central unit resources to coordinate information and operation technological equipment group activity. The controlling commands generated in structure are based on the data analysis collected from a CPS through the net. A big number in the structure of CPSs being monitored (a CPP huge segment) with a relatively simple topology requires a high-production calculator in the central unit, which simultaneously serve to several connections in parallel including the constant connection high-speed channel;

- the structure communication «bus», which is information processes integrator for equally valuable agents with multiplexed data transmission discipline. The bus structure feature is a simultaneous message decoding with all CPSs and the following traffic processing with only the CPS for which it was addressed. The data exchange in the structure is done in a mono-channel provided with the net coverage zone. The structure central unit in each moment of time could be only one of CPSs (any of them) to initiate the exchange;

- the structure communication «tree» is normally asymmetric and is characterized with the information exchange in the net segments with close or far action radius using external or internal 
(CPS integrated) concentrators and traffic routers. In the structure they use telecommunication protocols oriented for the information exchange in several alternative routes to increase the created connections reliability;

- the structure communication «cell» uses local net channels and gates to transform and transmit the data between the operation system earth segment and the CPP cloud platform. The structure supports the cell connection mechanisms to regulate the CPS wireless traffic. The data exchange is done with CPS in pairs with long radius net coverage. The original configuration installs randomly and then are corrected dynamically;

- the structure communication «hybrid» relates with nets with programmable configuration. The structure uses different configuration options of elementary topologies and connection transit channels. It supports information exchange protocols for heterogeneous nets with increased action radius, which are important for production and engineer CPSs hybrid control. The broad broadcast storm collision justified with radio ether information capacity are eliminated with restrictions imposed on connection protocols and re-translators number installed in the CPP sites.

\section{Conclusion}

Companies, which create a CPP today actively look for a way how to solve the net construction problem, which are tolerant to the time delays in the connection channel. The reached results are concentrated to implement CPSs specially designed for the IoT net, which function autonomously with individual algorithms. The net exchange in some CPSs are based on standard procedures (the line listening, awaiting for available connection and traffic transmission, confirmation that address agents have received the necessary messages) with subsequent connection sessions and idling periods in the given time slots.

The IoT net building oriented to control plant CPS devices including the designing of more complicated industrial equipment samples where the support operation and information processes functions are maximum-ly divided. Coordinator units elements to control the net must be reasonably placed directly as a part of CPS, which in some topologies are serviced with gates. Connection session made with a new generation CPS will automatically synchronize the equipment inner timer with the external net time according to which they transform information in the operation environment into the cloud applications data. This kind of information exchange let CPS not to release some additional traffic to the net containing controlling information (service one).

The CPS information exchange with the environment dynamic topology must be constructed with time logical addresses and physical constant addresses of equipment, which transmit and receive messages in a short time window. The connection interval continuance made by the local net designers including general access CPP nets must be defined with statistical data to characterize the average time of the safe package delivery. The industrial IoT segment containing a lot of broadcasting CPSs must be projected with the level of electromagnetic disturbances considered and how to protect the equipment from that. The message transport from the end CPS devices to the server and reverse, which must be done with traffic planning, which is transmitted through the gates to the transit nets and Internet.

The given topology options form the dynamically reconfigurable calculation net containing physical CPS devices with integrated software. The CPS interaction net space is an informative and communication including components of operation and cybernetic nature to unite heterogeneous connection channels into a single CPP platform. The data exchange combined mechanisms into static and dynamic net structures are done through the net gates to provide concord and work together for information protocols and connection technologies based on poly-model telecommunication interfaces.

The CPP information net topology details let form new industrial objects control models, which use CPSs. CPSs integration into a multi-loop system of automatic control is done with multi-domain CPS device representation connected to wired or wireless connection channel. To optimize the information tides they need a control server to distribute the CPP traffic among net agents. A multi-domain mode to describe a CPS into CPP information nets are done in program level of digital twins models to reflect physical industrial equipment into the electronic objects virtual environment space. 


\section{References}

[1] M. Helu, T. Sprock, D. Hartenstine, R. Venketesh, W. Sobel, CIRP Annals 69(1) (2020) 385-388.

[2] O. Salunkhe, O. Stensota, M. Akerman, A. F. Berglund, P-A. Alveflo, IFAC-PapersOnLine 52(13) (2019) 1632-1637.

[3] B. Diene, J. J. Rodrigues, O. Diallo, E. H. Ndoye, V. V. Korotaev, Mechanical systems and signal processing 138106564 (2020).

[4] Y. Ze, L. Liu, Z. Kun, Z. Jianhua, Procedia computer science 147 (2019) 145-150.

[5] A. V. Gurjanov, D. A. Zakoldaev, A. V. Shukalov, I. O. Zharinov, Journal of Physics: Conference Series 1059(1) 012010 (2018).

[6] M. H. Rehman, I. Yaqoob, K. Salah, M. Imran, P. P. Jayaraman, C. Perera, Future generation computer systems 99 (2019) 247-259.

[7] N. Muthukumar, S. Srinivasan, K. Ramkumar, D. Pal, J. Vain, S. Ramaswamy, Future generation computer systems 95 (2019) 354-363.

[8] A. V. Gurjanov, D. A. Zakoldaev, A. V. Shukalov, I. O. Zharinov, Journal of Physics: Conference Series 1015(5) (2018) 052035.

[9] J. Cheng, W. Chen, F. Tao, C-L. Lin, Journal of industrial information integration 10 (2018) 1019.

[10] F. Zhu, W. Wu, Y. Zhang, X. Chen, Information sciences 502 (2019) 218-228.

[11] A. Fast-Berglund, M. Åkerman, D. Li, O. Salunkhe, IFAC-PapersOnLine 52(13) (2019) 1525-30.

[12] B. Liu, Y. Zhang, G. Zhang, P. Zheng, Advanced engineering informatics 42100984 (2019).

[13] C. Kan, H. Yang, S. Kumara, Journal of manufacturing systems 46 (2018) 282-293.

[14] F. Zezulka, P. Marcon, Z. Bradac, J. Arm, T. Benesl, I. Vesely, IFAC-PapersOnLine 51(6) (2018) $150-155$.

[15] A. V. Gurjanov, D. A. Zakoldaev, A. V. Shukalov, I. O. Zharinov, IOP Conference Series: Materials Science and Engineering 327(2) 022111 (2018). 